\title{
Deep learning from HE slides to predict the clinical benefit from adjuvant chemotherapy in hormone receptor-positive breast cancer
}

\section{Soo Youn Cho}

Samsung Medical Center

Jeong Hoon Lee

Lunit inc.

Jai Min Ryu

Samsung Medical Center

Jeong Eon Lee

Samsung Medical Center

Eun Yoon Cho ( $\nabla$ eunyoon.cho@samsung.com )

Samsung Medical Center

Chang Ho Ahn

Lunit inc.

\section{Kyunghyun Paeng}

Lunit inc.

Inwan Yoo

Lunit inc.

Chan-Young Ock

Lunit inc.

\section{Sang Yong Song}

Samsung Medical Center

\section{Research Article}

Keywords: Deep-learning, HE, OncotypeDx, Recurrence Score, Adjuvant chemotherapy

Posted Date: December 10th, 2020

DOl: https://doi.org/10.21203/rs.3.rs-118010/v1

License: (c) (i) This work is licensed under a Creative Commons Attribution 4.0 International License.

Read Full License 


\section{Abstract}

Background: The predictive value of adjuvant chemotherapy for early-stage hormone receptor-positive breast cancer has been only validated by a 21-gene expression assay. We hypothesized that deeplearning prediction from HE images, called Lunit-SCOPE, is a potential prognostic and predictive biomarker of adjuvant chemotherapy.

Methods: We retrospectively collected HE slides from 1153 de-identified breast cancer patients at the Samsung Medical Center (SMC) in order to develop a deep-learning algorithm called Lunit-SCOPE. The histological parameters from 255 patients, deciphered by Lunit-SCOPE, were trained to predict the recurrence score (RS) using the 21-gene assay from Oncotype DX. We validated the model's performance using the recurrence survival of 898 patients and The Cancer Genome Atlas (TCGA) cohort, and examined related biological functions through RNA sequence data.

Results: The histologic parameter-based RS prediction model predicted the oncotype DX score $\left(\mathrm{R}^{2}=0.96\right)$ and the recurrence survival analysis on the validation $(p<0.01)$ and TCGA cohort $(p<0.01)$, where the most important variables were the nuclear grade and the mitotic cells in the cancer epithelium. Of the 85 patients classified as the high-risk group, 72 patients who received adjuvant therapy had a significantly better survival $(p<0.01)$. The functions of the top 300 highly correlated genes with a predicted RS were enriched for cell cycle, nuclear division and cell division. Of the 21-genes from the Oncotype DX, the predicted RS had positive correlations with the proliferation category genes and was negatively correlated with the prognostic genes in the estrogen category.

Conclusion: An integrative analysis using Lunit-SCOPE predicts a high risk of recurrence and those who would benefit from adjuvant chemotherapy for early-stage hormone-positive breast cancer.

\section{Introduction}

Breast cancer is the most common cancer in women worldwide, and hormone-receptor (HR)-positive, lymph node-negative diseases account for nearly half of all the breast cancer cases ${ }^{1,2}$. As the role of adjuvant chemotherapy (ACTx), in addition to hormonal therapy, in this subgroup has been controversial over the last decade, the use of gene expression profiling for assessing the risk of recurrence and the potential benefit from ACTx has emerged. There are many multigene assays for assessing the prognosis of HR-positive, lymph node-negative, early-stage breast cancer ${ }^{3-6}$. However, only the 21-gene assay (Oncotype Dx) has proven its significance with respect to the clinical guidance of ACTx according to the recurrence score (RS) ${ }^{7,8}$.

Although, despite the well-proven clinical utility of RS for the 21-gene assay, its effectiveness in all patients with HR-positive, lymph node-negative, early-stage breast cancer is still controversial along with its cost burden in countries outside of the US ${ }^{9,10}$. Moreover, the instability of handling RNA extracted from formalin-fixed paraffin embedded (FFPE) tissue in real-world practice might compromise its 
accuracy and interfere with the appropriate translation of the RS result ${ }^{11}$. Therefore, a more simple and efficient method for assessing RS, using permanent tissue, is mandatory. Since the RS from the 21-gene assay is mainly due to the proliferation group score (MKI67, STK15, BIRC5, CCNB1, and MYBL2) and the mitotic count is clearly associated with the RS ${ }^{7}$, a comprehensive pathologic examination, including mitosis and other features of the cell-cell interactions, consistently reflects the RS.

Thus, we developed a deep learning (DL)-based HE image analyzer called a Lunit-SCOPE, which identifies and quantifies various histologic parameters from HE-stained whole slide images (WSI). Previously, the Lunit-SCOPE was shown to accurately detect tumor cells and other cells in the microenvironment, and it also clearly predicted mitosis in each cell in breast cancer ${ }^{12}$. Data reveal that The Cancer Genome Atlas (TCGA) pan-cancer analysis, Lunit-SCOPE predicts an abundance of cancer-associated stroma in pancreatic adenocarcinoma and a consensus of molecular subtype 4 of colon cancer ${ }^{13}$, as well as highly-inflamed tumor-infiltrating lymphocytes in immunogenic tumors, such as renal cell carcinoma, melanoma, and urothelial cancer ${ }^{14}$.

Since Lunit-SCOPE accurately reflects the comprehensive features of HE slides, especially with regard to mitotic count and the infiltration of immune cells or stromal cells, we hypothesized that histological parameters analyzed by Lunit-SCOPE would predict the RS from the 21-gene assay, revealing a potential prognostic and predictive biomarker of ACTx in early-stage hormone receptor-positive breast cancer.

\section{Results}

\section{Detection of various cell types in the breast cancer HE slides}

The Lunit-SCOPE deciphers an HE slide image into histologic parameters through three panels, including the tissue, structure, and cell panel. The process to develop the Lunit-SCOPE and the workflow scheme of this study are illustrated in Fig. 1 (detailed description in the supplementary methods). Each panel is an independent multi-class prediction model trained through curated ground truth annotations by expert pathologists. The panels decipher the histologic parameters in the image divided into small patch images and finally returns the aggregated count values corresponding to the tissue, structure, and cell from the WSIs. The performance of the three panels is described in Supplementary Table 1.

\section{Development of a model to predict RS based on histologic parameters}

The study included a total of 1704 patients with HE-stained WSIs and clinical information, including recurrence and survival (Table 1). A total of 255 patients with a 21-gene assay score by Oncotype DX were used as a training dataset to predict the RS using the histologic parameters derived by Lunit-SCOPE. The internal validity of the trained 21-gene assay was 3.67 for the root of the MSE, 2.65 for the mean absolute value (MAE), and 0.96 for the Pearson correlation coefficients with the raw 21-gene assay score. The trained model identified 31 high-risk patients when the two groups were divided based on a score of 20 , which was the usual beneficial group whose potential benefit for chemotherapy compared to the risk 
of side effects. The recurrence survival of both of the groups differed significantly in the log-rank $(p<$ 0.001 ) and cox proportional hazard models $(p=0.002, \mathrm{HR}=6.43)$. (Fig. $2 \mathrm{~A})$.

Table 1

Clinical characteristics of the hormone receptor-positive breast cancer patients for the model development cohort, the SMC validation cohort and the TCGA BRCA cohort.

\begin{tabular}{|c|c|c|c|}
\hline & Model development & SMC Validation & TCGA BRCA \\
\hline No. & 255 & 898 & 551 \\
\hline Age & $45.89(7.83)$ & $53.34(1.92)$ & $59.98(13.30)$ \\
\hline Sex & 255 & 898 & 544 \\
\hline Female & 0 & 0 & 7 \\
\hline \multicolumn{4}{|l|}{ Male } \\
\hline Stage & 248 & 636 & 102 \\
\hline I & 3 & 246 & 268 \\
\hline II & 0 & 16 & 113 \\
\hline III & 0 & 0 & 0 \\
\hline \multicolumn{4}{|l|}{ IV } \\
\hline Subtype & 255 & 889 & 475 \\
\hline $\mathrm{ER}+$ & 245 & 843 & 415 \\
\hline $\mathrm{PR}+$ & 0 & 0 & 0 \\
\hline \multicolumn{4}{|l|}{ HER2+ } \\
\hline AdjCTx & 33 & 535 & - \\
\hline Yes & 222 & 363 & - \\
\hline \multicolumn{4}{|l|}{ No } \\
\hline AdjHTx & - & 868 & - \\
\hline Yes & - & 30 & - \\
\hline \multicolumn{4}{|l|}{ No } \\
\hline Oncotype DX score & $15(11-19)$ & - & - \\
\hline Follow-up years & $2.28(1.96-4.10)$ & $9.13(8.17-9.92)$ & $1.93(0.46-3.06)$ \\
\hline
\end{tabular}


The top 20 important histologic parameters to predict the RS based on 21-gene assays are listed in the variable importance barplot (Fig. 2B). The most important variable for predicting the RS of 21-gene assay was the mitotic cell count located in the cancer epithelium, followed by nuclear grades 1 and 2 . The remaining important features can be found in Supplementary Table 2. Nine of the top 10 important variables were all in the cancer epithelium and cancer stroma domains. The other histologic parameters that were not included in the list were lowly counted values, which were filtered out in the histologic parameter preprocessing step.

\section{Clinical validation of predicted RS in an independent cohort}

The RS values of 898 SMC cohort and 551 TCGA cohort were produced by Lunit-SCOPE to validate our model. The medians for the predicted RS of the SMC model development and validation cohort were 17.24 and 16.00, respectively (Supplementary Fig. 1). The time to disease recurrence and survival analysis by the threshold 20 of the predicted RS was performed on both cohorts. Patients predicted to be the high-risk group had a significantly poorer survival than the low-risk patients $(p<0.01, H R=2.26)$ (Fig. 3A). In the multivariate cox proportional hazard model, with clinical variables, the predicted RS was most significant $(p<0.01)$, with a 2.29 hazard ratio for the high risk group followed by the T-stage, age, $\mathrm{N}$ stage and treatment of adjuvant chemotherapy. The details of the multivariate and univariate cox proportional hazard model for the disease-free survival (DFS) in SMC validation can be found in Supplementary Table 3.

The predicted RSs from the 551 TCGA breast cancer data were used as the external validation set. Survival rate of the TCGA cohort was worse than that of the SMC cohort $(p<0.001)$, and the median of predicted RS of the TCGA cohort was higher than that of the SMC cohort. Among the 187 high-risk patients with predicted RS, more than 20 showed worse significantly prognoses compared to the low-risk patients $(p=0.003)$, in spite of the fact that these patient were of more advanced stages (Fisher's exact test, $p=0.024)$ (Fig. 3B).

ACTx was recommended for the patients defined as the high-risk group, which was over 20 in the RS patients from the 21-gene assay, and we assessed the DFS for the predicted RS using over 20 patients with ACTx to confirm the utility of our model. In 363 patients who did not received ACTx, the high-risk patients showed a worse survival than the low-risk patients $(p<0.0001)$ (Fig. 3C). However, for the 535 patients who received ACTx, there was no difference between the two risk groups for prognosis according to the predicted RS $(p=0.37)$ and the multivariate analysis with age, T-stage, and $\mathrm{N}$-stage as well $(p=$ 0.323) (Supplementary Fig. 2). Then, we divided all the patients into four groups by their ACTx status and a threshold of 20 of predicted RS (Fig. 3D). The log-rank p-value for the survival analysis of the four groups showed a significantly $(P<0.001)$ worse prognosis in the high-risk patients without ACTx. There was no statistically significant difference for recurrence and survival $(p=0.104)$ among other three groups. The clinical characteristics of the four groups divided by the predicted RS and the adjuvant treatment are summarized in Supplementary Table 4.

\section{Distinct genomic and transcriptomic characteristics of the predicted RS in TCGA}


We analyzed the TCGA cohort gene expression data associated with the predicted RS using 551 pathology images. The top 300 genes that had the highest correlation coefficient with the predicted RS were used for the functional enrichment analysis of the BP, CC and MF for the Gene Ontology and KEGG pathways. Based on the Bonferroni-corrected significance threshold $(p<0.05), 151$ significant Gene Ontology and KEGG pathway terms were identified. The top 20 functional terms and pathways are shown in Fig. 4, with the negative log2 based p-values. Mitotic cell cycle, cell cycle, nuclear division, and cell division were enriched biological process functions in the Gene Ontology for the top 300 genes. Among the various cellular parameters, spindle and chromosome, which play an important role in the cell cycle, were significantly enriched. With regard to molecular function, protein binding was significantly enriched. The cell cycle was also a significant term in the KEGG pathways. The details of the functional terms, genes, and the significance of the 151 functions are available in Supplementary Table 5.

Of the 21 genes used in the Oncotype Dx test, 16 genes, excluding the reference gene, were analyzed for correlation with the predicted RS and were ordered by correlation coefficient (Table 2). All the genes in proliferation category, including AURKA, MKI67, MYBL2, BIRC5, and CCNB1, were positively correlated with the predicted RS, and the estrogen receptor genes, including ESR1, PGR, SCUBE2 and BCL2, were negatively correlated. The other genes in the other categories, including invasion and HER2, had significantly lower correlations than those in the proliferation and estrogen receptor categories (Wilcoxon rank sum test, $p=0.003, p=0.006$ ). 
Table 2

Correlation between the predicted RS and the genes in the Oncotype DX gene assay.

\begin{tabular}{|llll|}
\hline Genes & Cor. & p.value & Category \\
\hline AURKA & 0.260 & $7.88 \mathrm{E}-10$ & proliferation \\
\hline MKI67 & 0.250 & $3.37 \mathrm{E}-09$ & proliferation \\
\hline MYBL2 & 0.249 & $3.77 \mathrm{E}-09$ & proliferation \\
\hline BIRC5 & 0.208 & $1.02 \mathrm{E}-06$ & proliferation \\
\hline CCNB1 & 0.196 & $4.22 \mathrm{E}-06$ & proliferation \\
\hline CTSL2 & 0.146 & $6.34 \mathrm{E}-04$ & Invasion \\
\hline CD68 & 0.132 & $2.00 \mathrm{E}-03$ & other genes \\
\hline MMP11 & 0.094 & $2.80 \mathrm{E}-02$ & Invasion \\
\hline ERBB2 & 0.005 & $8.99 \mathrm{E}-01$ & HER2 \\
\hline GRB7 & 0.005 & $9.08 \mathrm{E}-01$ & HER2 \\
\hline GSTM1 & 0.000 & $9.94 \mathrm{E}-01$ & other genes \\
\hline BAG1 & -0.090 & $3.55 \mathrm{E}-02$ & other genes \\
\hline ESR1 & -0.135 & $1.58 \mathrm{E}-03$ & Estrogen \\
\hline PGR & -0.138 & $1.23 \mathrm{E}-03$ & Estrogen \\
\hline SCUBE2 & -0.164 & $1.30 \mathrm{E}-04$ & Estrogen \\
\hline BCL2 & -0.216 & $3.75 \mathrm{E}-07$ & Estrogen \\
\hline
\end{tabular}

\section{Discussion}

We developed a DL-based HE image analyzer called Lunit-SCOPE, in order to identify and quantify various histologic parameters from HE-stained whole slide images. Using the pathology features derived from Lunit-SCOPE, we developed a prediction model for the 21-gene assay RS from Oncotype DX, revealing potential prognostic and predictive biomarkers of ACTx in early-stage HR-positive breast cancers, including SMC and TCGA cohorts. Importantly, for the patients without ACTx, the patients predicted as high-risk through the predicted RS were significantly worse, and the patients with ACTx in the high-risk group had a significantly better prognosis. Moreover, a gene set enrichment analysis biologically validated that the predicted RS was associated with pathways involved in the cell cycle and nuclear grade, which are related with a high risk of recurrence. 
Recent advances in DL analysis shed a light on novel approach to understand cancer biology. Growing evidence shows that DL analyses of medical images are clinically reliable tools for diagnosis ${ }^{15-17}$. However, the clinical significance of this technology for a predictive biomarker has not been reported yet. Lunit-SCOPE was developed using $>1,000$ breast cancer slides, annotated various cell types, and tissue architectures. The preliminary results showed that Lunit-SCOPE accurately predicted tumor proliferation in breast cancer, and a core biologic explanation for how the 21-gene expression assay works in the prediction of high-risk patients would be by evaluating proliferation genes ${ }^{12}$. Moreover, Lunit-SCOPE also detects cancer-associated fibroblasts that dirupt the stromal barrier and induce the infiltration of tumorassociated macrophages ${ }^{18,19}$, which reflects cancer aggressiveness. Therefore, we hypothesized that Lunit-SCOPE would be able to predict high-risk patients who would benefit from ACTx with clinical significance.

The 21-gene expression assay test includes the gene categories proliferation, estrogen, HER2, invasion, and other cancer-related genes. The five genes related to cancer proliferation had a positive correlation with the predicted RS, based on Lunit-SCOPE, with the pathology images. This suggests that the expression of the genes related to proliferation, cell cycle, and progression ultimately affected the components of the pathology image, which are related to recurrence. All four genes in the estrogen category also had negative correlations with the predicted RS. The Progesterone Receptor $(P G R)$ and Estrogen Receptor 1 (ESR1) are well known genes that influence cancer recurrence. The apoptosis regulator BCL2 was also negatively correlated and is a gene known as a favorable prognostic marker. The directionality of the correlations between the expressions of the recurrence-related genes and the predicted RS indicates that the prediction of this model based on the pathology was consistent with that of the 21-gene expression assay.

There are several limitations in the current study. Firstly, the RS of the training cohort did not have range that was enough to predict RS. As recent clinical trials show that endocrine treatment alone is not inferior to endocrine treatment plus chemotherapy in patients with RS 11 through 25, the most well-validated cutoff of RS for the decision of adding chemotherapy would be $25^{8}$. However, in the current study, only a few patients in training cohort had an RS $>25$, and the predicted RS of the training or validation cohort was also dispersed in range that was to the ground truth RS. Therefore, we cannot fully ascertain that this model accurately predicts the RS $>25$. Nevertheless, we have shown that a high predicted RS was significantly correlated with a poor prognosis in patients who did not receive ACTx. Another limitation is the selection bias present in retrospective analysis, as patients who did not receive the chemotherapy were related to other clinical factors, such as a poor performance status or poor compliance. Moreover, physicians would choose patients that are clinically high-risk to receive ACTx. This factor could contribute to the worse clinical outcome in the patients with ACTx compared to those without ACTx. To overcome this limitation, a well-designed prospective clinical trial is warranted.

In conclusion, an integrative analysis of Lunit-SCOPE predicts a high risk of recurrence, as well as who would benefit from adjuvant chemotherapy by predicting the RS of the 21-gene expression array in earlystage hormone-positive breast cancer. 


\section{Materials And Methods}

\section{Patients and tumor tissues for the pathology slides}

The protocol for this retrospective study was approved by the Ethics Committee of the Institutional Review Board (IRB 2018-03-038-002) and informed consent was obtained from all subject at the Samsung Medical Center (SMC). All experiments were performed in accordance with relevant guidelines and regulations, all methods were carried out in accordance with relevant guidelines and regulations, and all experimental protocols were approved by SMC. A total of 1153 pathology slide images, derived from anonymized HE-stained tissue samples from breast cancer patients with histologically confirmed hormone receptor-positive tumors, were aquired using a WSI scanner (Pannoramic 1000, 3DHISTECH Ltd) at the magnification of 40X. Among them, 255 images of the patients with 21-gene assay scores from Oncotype DX ${ }^{\mathrm{TM}}$ (Genomic Health) were used to develop the model, and the remaining 898 images were used as a validation cohort to confirm the prognostic and predictive values of the predicted RS of the 21gene assay scores. A total of 551 cases, with both digital pathology images and image-matched RNA sequence data from primary tumor tissues, were included in the data analysis. The TCGA breast cancer patients with HR-positive and human epidermal growth factor receptor-2 (HER2) negative, with the exclusion of advanced stage patients, were used for the external validation for the assessment of the prognostic significance ${ }^{20}$.

\section{Development of the DL model}

For the training, anonymized HE-stained tissue slides were reviewed by expert pathologists (SYC, EYC and SYS). The informative regions from these slides were manually selected and annotated by the expert pathologists. Then, we trained convolutional neural networks (CNNs) to decipher various types of histologic parameters ${ }^{21}$. The WSIs were tiled into $50 \%$ overlapped $4096 \varangle 4096$ patches to analyze and quantify the histologic parameters. The performance for these models was evaluated using the validation set with the accuracy, intersection over union (IoU), and mean average precision (mAP) with distance.

\section{Raw count of the histologic parameter preprocessing}

The count from the histologic parameters that were quantified by Lunit-SCOPE had a count distribution according to the tissue, structure, and cell types. We applied the Trimmed Mean of M-values (TMM) count normalization for the histologic parameters count to make accurate comparisons of the data proportions between the samples without missing the data composition ${ }^{22}$.

\section{TCGA RNA sequencing data analyses}

The RNA-seq data for breast cancers were obtained from the TCGA Broad Institute GDAC Firehose. The RNA sequencing raw count samples, quantified by RNA-seq by expectation (RSEM), were used ${ }^{23}$. To filter out the genes with low expression levels, the genes with counts per million (cpm) values $<1$ in at least half of the samples were excluded ${ }^{24}$. The raw read counts were normalized by the TMM and the logCPM transformation with limma voom. Finally, the expressions of 17,649 genes were used for this analysis ${ }^{25}$. 
To find the biological function associated with the predicted RS from the 21-gene assay, we performed a Pearson correlation analysis with the gene expression. The top 300 high correlated genes were selected as related genes, and an enrichment analysis was performed for the Biological Process (BP), Cellular Component (CC) and Molecular Function (MF) terms in the Gene Ontology and KEGG Pathway database using the RDAVIDWebService tool in Bioconductor ${ }^{26-28}$.

\section{Prediction of RS using random forest (RF) regression}

Fast unified RFs for survival, regression, and classification (RF-SRC), non-parametric statistical estimation, was used to predict the RS from the 21-gene assay based on Lunit-SCOPE ${ }^{29}$. Since the RS from the 21-gene assay in the validation set is not available for ground truth, the RF model that can be trained with the OOB training data itself was selected. The method provides an original variable's importance index for regression with the reprioritization component of the RS assessments. The model was developed using bootstrap samples with the RS, and the Out-of-Bag (OOB) samples were used as test samples. A variable's importance was defined as the mean decrease in the tree's performance for the randomly permuted OOB samples. The loss function for minimizing the mean squared error (MSE) was used for the model assessment metrics in the regression problem along with the coefficients of determination $\left(R^{2}\right)$ to assess the RF regression's goodness-of-fit and predictive performance of the RS from the 21-gene assay.

\section{Declarations}

\section{AUTHOR CONTRIBUTION}

S.Y. Song designed and organized the experiment.

S.Y. Cho and J.H. Lee led the integrative analyses.

S.Y. Cho, E.Y. Cho and S.Y. Song performed the integrative analysis of the pathology slides.

J.H. Lee, C.H. Ahn, K. Paeng, I. Yoo, and C.-Y. Ock performed and translated the deep learning analysis.

S.Y. Cho, J.H. Lee, and C.-Y. Ock wrote the initial draft. E.Y. Cho, C.H. Ahn, K. Paeng, I. Yoo, S.Y. Song revised the draft.

All the authors read and approved the final manuscript.

\section{ACKNOWLEDGEMENT}

This research was supported by Lunit Inc. We appreciated the patients and their families who generously donated their tissues to TCGA/TCIA, as well as the members of TCGA/TCIA who collected and disclosed the valuable data.

\section{Author Disclosure Statement}

Page 10/16 
J.H. Lee, C.H. Ahn, K. Paeng, I. Yoo, and C.Y Ock are Employees of Lunit.

Other authors declare no conflicts of interest.

Funding source: This research is funded by Lunit Inc.

\section{References}

1. Jemal, A., Center, M. M., DeSantis, C. \& Ward, E. M. Global patterns of cancer incidence and mortality rates and trends. Cancer Epidemiol. Prev. Biomarkers 19, 1893-1907 (2010).

2. Howlader, N. et al. US incidence of breast cancer subtypes defined by joint hormone receptor and HER2 status. JNCl J. Natl. Cancer Inst. 106, (2014).

3. Paik, S. et al. A multigene assay to predict recurrence of tamoxifen-treated, node-negative breast cancer. N. Engl. J. Med. 351, 2817-2826 (2004).

4. Van De Vijver, M. J. et al. A gene-expression signature as a predictor of survival in breast cancer. $N$. Engl. J. Med. 347, 1999-2009 (2002).

5. Lænkholm, A.-V. et al. PAM50 risk of recurrence score predicts 10-year distant recurrence in a comprehensive Danish cohort of postmenopausal women allocated to 5 years of endocrine therapy for hormone receptor-positive early breast cancer. J. Clin. Oncol. 36, 735-740 (2018).

6. Sestak, I. et al. Comparison of the performance of 6 prognostic signatures for estrogen receptorpositive breast cancer: a secondary analysis of a randomized clinical trial. JAMA Oncol. 4, 545-553 (2018).

7. Paik, S. et al. Gene expression and benefit of chemotherapy in women with node-negative, estrogen receptor-positive breast cancer. J Clin Oncol 24, 3726-3734 (2006).

8. Sparano, J. A. et al. Adjuvant chemotherapy guided by a 21-gene expression assay in breast cancer. N. Engl. J. Med. 379, 111-121 (2018).

9. Wang, S.-Y. et al. Cost-Effectiveness Analyses of the 21-Gene Assay in Breast Cancer: Systematic Review and Critical Appraisal. J. Clin. Oncol. Off. J. Am. Soc. Clin. Oncol. 36, 1619-1627 (2018).

10. Reed, S. D., Dinan, M. A., Schulman, K. A. \& Lyman, G. H. Cost-effectiveness of the 21-gene recurrence score assay in the context of multifactorial decision making to guide chemotherapy for early-stage breast cancer. Genet. Med. 15, 203 (2013).

11. Macabeo-Ong, M. et al. Effect of duration of fixation on quantitative reverse transcription polymerase chain reaction analyses. Mod. Pathol. 15, 979 (2002).

12. Paeng, K., Hwang, S., Park, S. \& Kim, M. A unified framework for tumor proliferation score prediction in breast histopathology. in Deep Learning in Medical Image Analysis and Multimodal Learning for Clinical Decision Support 231-239 (Springer, 2017).

13. Guinney, J. et al. The consensus molecular subtypes of colorectal cancer. Nat. Med. 21, 1350 (2015).

14. Paeng, K. et al. Pan-cancer analysis of tumor microenvironment using deep learning-based cancer stroma and immune profiling in H\&E images. (2019). 
15. Nam, J. G. et al. Development and validation of deep learning-based automatic detection algorithm for malignant pulmonary nodules on chest radiographs. Radiology 290, 218-228 (2018).

16. Hwang, E. J. et al. Deep Learning for Chest Radiograph Diagnosis in the Emergency Department. Radiology 191225 (2019).

17. Hwang, E. J. et al. Development and Validation of a Deep Learning--Based Automated Detection Algorithm for Major Thoracic Diseases on Chest Radiographs. JAMA Netw. open 2, e191095e191095 (2019).

18. Cid, S. et al. Prognostic influence of tumor stroma on breast Cancer subtypes. Clin. Breast Cancer 18, e123--e133 (2018).

19. Mahmoud, S. M. A. et al. Tumour-infiltrating macrophages and clinical outcome in breast cancer. J. Clin. Pathol. 65, 159-163 (2012).

20. Weinstein, J. N. et al. The cancer genome atlas pan-cancer analysis project. Nat. Genet. $\mathbf{4 5 , 1 1 1 3}$ (2013).

21. Tan, M. \& Le, Q. V. EfficientNet: Rethinking Model Scaling for Convolutional Neural Networks. arXiv Prepr. arXiv1905.11946 (2019).

22. Robinson, M. D. \& Oshlack, A. A scaling normalization method for differential expression analysis of RNA-seq data. Genome Biol. 11, R25 (2010).

23. Li, B. \& Dewey, C. N. RSEM: accurate transcript quantification from RNA-Seq data with or without a reference genome. BMC Bioinformatics 12, 323 (2011).

24. Robinson, M. D., McCarthy, D. J. \& Smyth, G. K. edgeR: a Bioconductor package for differential expression analysis of digital gene expression data. Bioinformatics 26, 139-140 (2010).

25. Smyth, G. K., Ritchie, M., Thorne, N. \& Wettenhall, J. LIMMA: linear models for microarray data. In Bioinformatics and Computational Biology Solutions Using R and Bioconductor. Statistics for Biology and Health. (2005).

26. Fresno, C. \& Fernández, E. A. RDAVIDWebService: a versatile R interface to DAVID. Bioinformatics 29, 2810-2811 (2013).

27. Ashburner, M. et al. Gene ontology: tool for the unification of biology. Nat. Genet. 25, 25 (2000).

28. Kanehisa, M., Furumichi, M., Tanabe, M., Sato, Y. \& Morishima, K. KEGG: new perspectives on genomes, pathways, diseases and drugs. Nucleic Acids Res. 45, D353-D361 (2016).

29. Ishwaran, H., Kogalur, U. B., Blackstone, E. H., Lauer, M. S. \& others. Random survival forests. Ann. Appl. Stat. 2, 841-860 (2008).

\section{Figures}




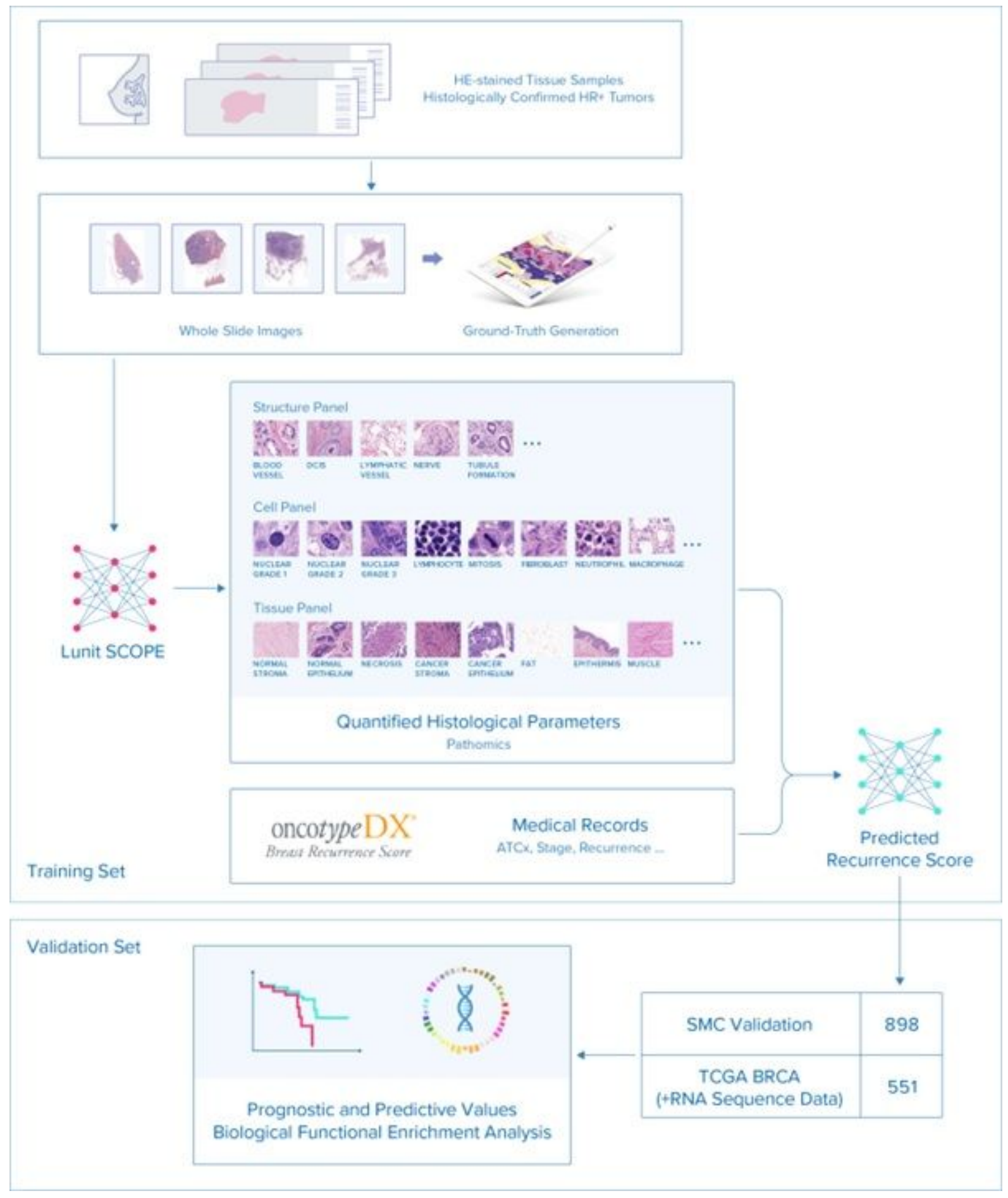

\section{Figure 1}

The scheme of the development of the Lunit-SCOPE and the workflow scheme of this study. 
A

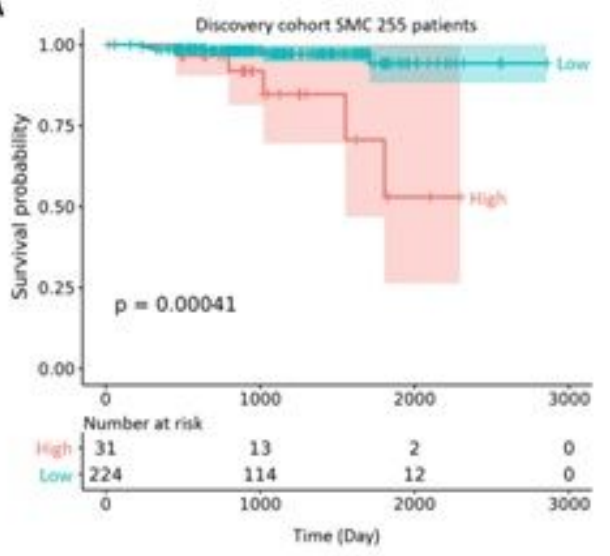

C

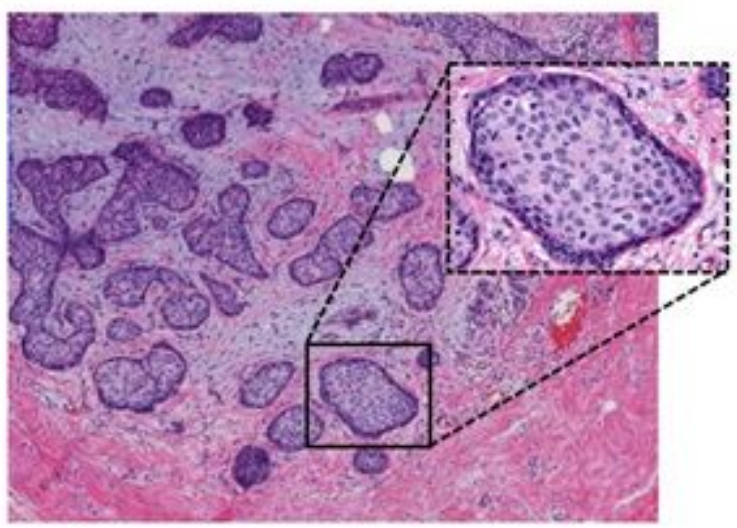

B

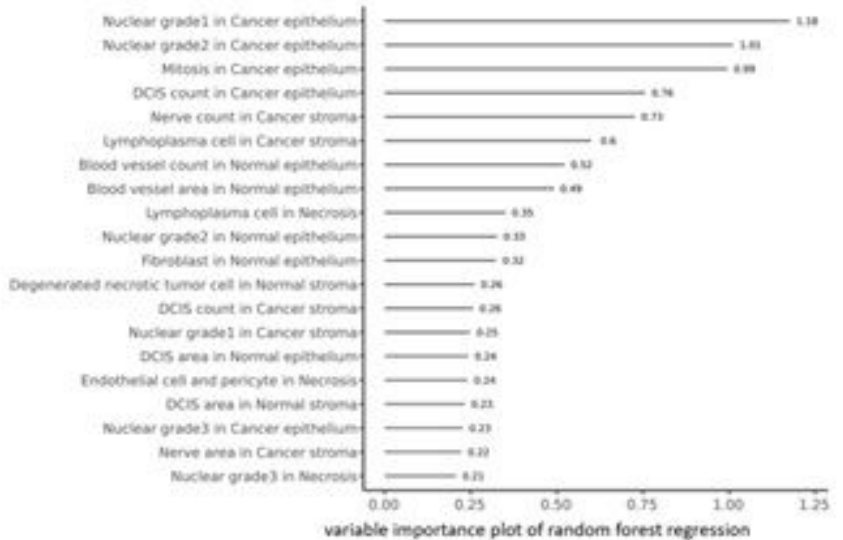

D

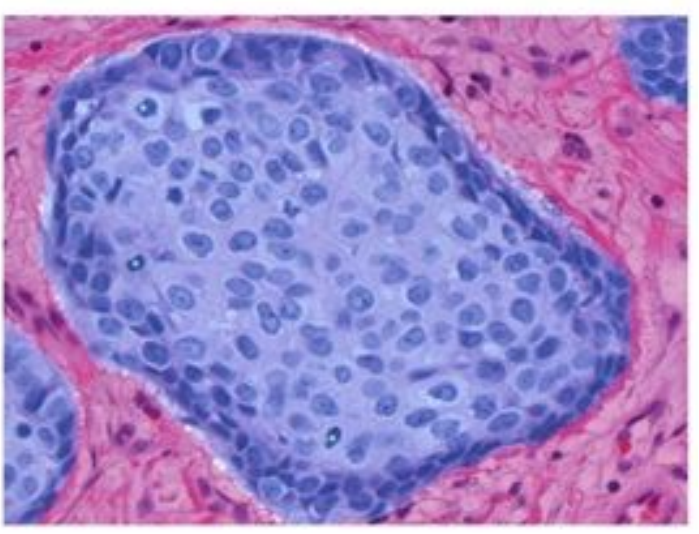

\section{Figure 2}

DFS analysis of the model and important features to predict te RS of OncotypeDX. a) The groups were divided by the prediction threshold of +20 , which was derived from the OncotypeDX prediction model, and were significantly different for the DFS. b) The top 20 important pathological parameters to predict the OncotypeDX score. c) WSI patch of high-risk patients and highlighted epithelium. d) Segmented regions for cancer epithelium region and detected mitotic cells by Lunit-SCOPE (Cyan). 
A
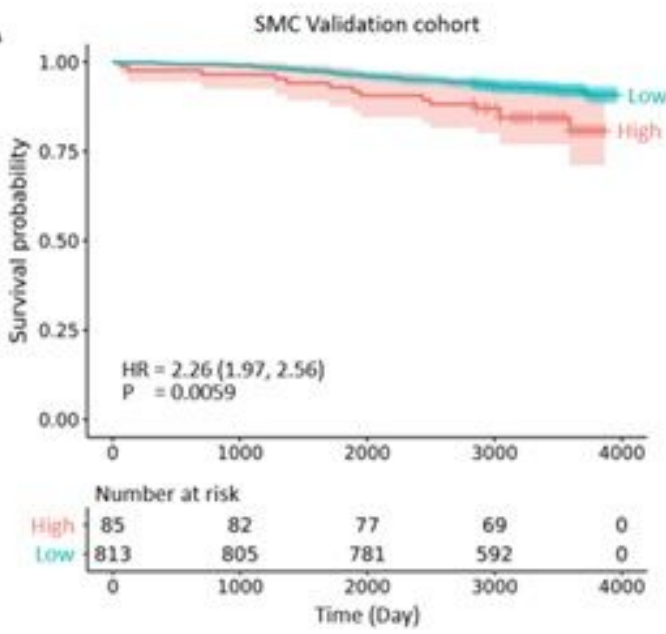

C

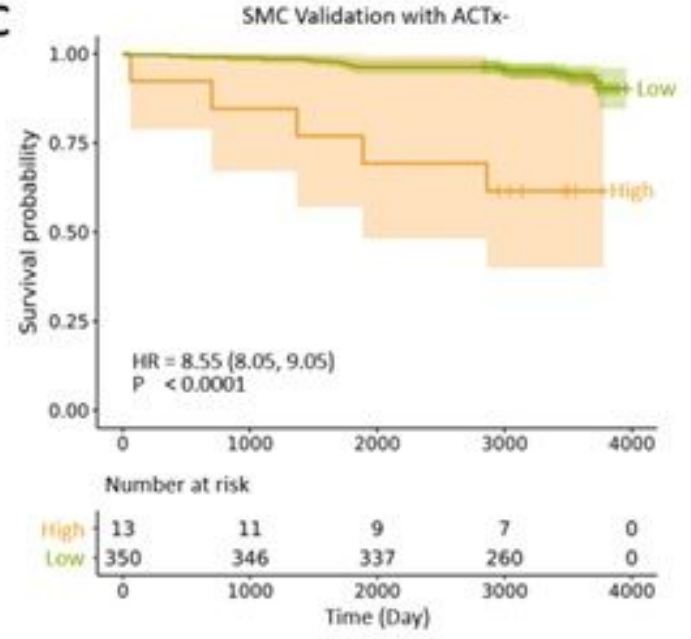

B
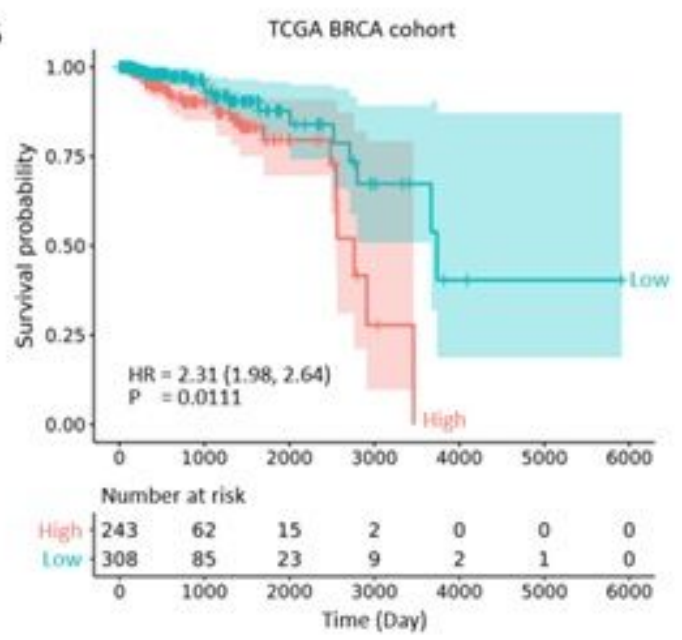

D

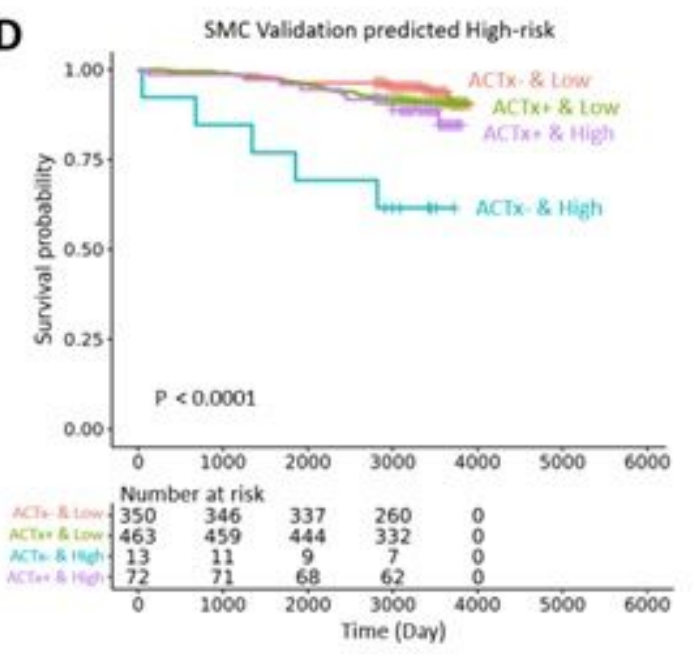

\section{Figure 3}

Time to disease recurrence survival analysis using a threshold of 20 from the predicted RS was performed using the SMC validation cohort and the TCGA BRCA cohort. a) The overall patient DFS was divided into two groups based on the predicted OncotypeDX of threshold 20 in the SMC validation cohort, and b) External validation of the RS prediction model in the TCGA dataset. c) DFS for the RS in the patients without adjuvant chemotherapy treatment. d) DFS of the patients by the RS prediction and adjuvant chemotherapy treatment. 


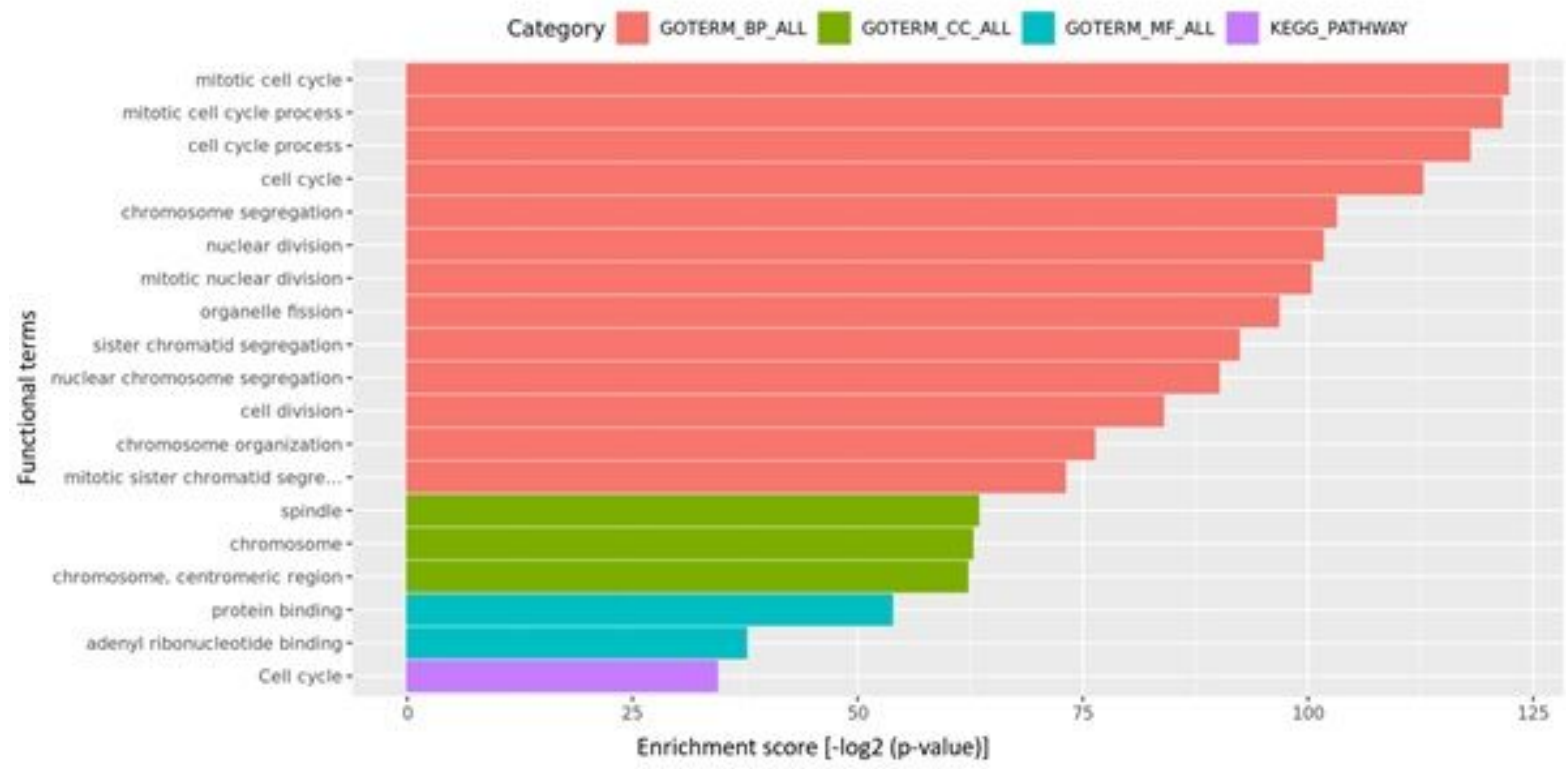

Figure 4

Functional enrichment analysis of the top 300 correlated genes with the predicted RS in the TCGA BRCA cohort

\section{Supplementary Files}

This is a list of supplementary files associated with this preprint. Click to download.

- SFigure1.png

- SFigure2.png

- SupplementaryTables.pdf

- SupplementaryMethods.pdf 